\title{
Immunohistochemical study of NF-kB p65, c-IAP2 and caspase-3 expression in cervical cancer
}

\author{
LI XIA and XIU-ZHEN XUE
}

The First Affiliated Hospital of Henan Scientific and Technological University, Henan, Luoyang 471003, P.R. China

Received October 20,2011; Accepted December 28, 2011

DOI: $10.3892 / \mathrm{ol} .2012 .564$

\begin{abstract}
The present study aimed to evaluate the expression of NF- $\kappa \mathrm{B}$ p65, c-IAP2 and caspase-3 in human cervical cancer. Immunohistochemistry was performed to determine the levels of NF- $\mathrm{B}$ p 65, c-IAP2 and caspase-3 expression in cervical cancer. Our study found overexpression of NF- $\kappa \mathrm{B}$ p65 and c-IAP2 and reduced expression of caspase- 3 in cervical cancer and cervical intraepithelial neoplasia (CIN) II-III. The expression of these molecules had no significant differences in the various clinical stages of cervical cancer, but the expres-

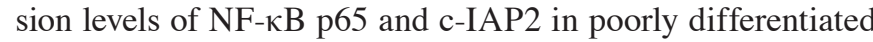
tumors and metastatic lymph nodes were higher than levels in well-differentiated tumors and non-metastatic lymph nodes. By contrast, caspase-3 expression in well-differentiated tumors and non-metastatic lymph nodes was higher than that in poorly differentiated tumors and metastatic lymph nodes. Overexpression of $\mathrm{NF}-\kappa \mathrm{B}$ p 65 and c-IAP2 and reduced expression of caspase- 3 in cervical cancer may be important in the tumorigenesis and development of cervical cancer, and they may be useful as biomarkers to estimate progression of cervical cancer.
\end{abstract}

\section{Introduction}

Cervical cancer is the most common cancer to develop in women. Although in recent years, great progress has been achieved in the early diagnosis of cervical cancer, its incidence and mortality remain high, and there is an overall trend that the age of onset for cervical cancer is decreasing. At present, few diagnostic and prognostic biomarkers for cervical cancer are in clinical use; therefore, recently, more attention has been increasingly paid on exploring novel biomarkers for the diagnosis and prognosis of cervical cancer, which involves translational research in the clinic based on the knowledge gained though basic research.

Correspondence to: Dr Xiu-Zhen Xue, The First Affiliated Hospital of Henan Scientific and Technological University, Henan, Luoyang 471003, P.R. China

E-mail: xuexzh@126.com

Key words: NF-кB p65, с-IAP2, caspase-3, cervical cancer, immunohistochemistry
Previously, research studies on nuclear factor $-\kappa \mathrm{B}(\mathrm{NF}-\kappa \mathrm{B})$ focused on inflammation and the immune response. Recent studies have found that $\mathrm{NF}-\kappa \mathrm{B}$ is also important in oncogenesis and tumor development. The upregulation of $\mathrm{NF}-\kappa \mathrm{B}$ was presented in a variety of epithelium-oriented cancers. A number of studies have reported that $N F-\kappa B$ promotes oncogenesis, tumor cell proliferation and invasion/metastasis through machinery of transcriptional regulation of the corresponding gene. Apoptosis inhibitory protein-2, also known as inhibitor of apoptosis protein-2 (c-IAP2), a member of the anti-apoptotic family of proteins (IAPs) is a potent anti-apoptotic factor which inhibits apoptosis through its interaction with a variety of cytokines. It has been reported that c-IAP2 suppresses tumor necrosis factor (TNF)-induced cell death under NF- $\mathrm{NB}$ control. Caspases or cysteine-aspartic proteases are cysteine-dependent aspartate-directed proteases that are essential in apoptosis, a mechanism of programmed cell death independent of apoptotic protease-mediated cleavage. Since caspases share two specific characteristics, cysteine protease and aspartic acid cleavage sites, they are also known as the caspase protease family (1). Caspase- 3 is the most important member of the caspase family and is closely related to the apoptotic process in eukaryotic cells. It is also involved in cytokine maturation, cell growth and differentiation. Based on our knowledge concerning the clinicopathological significance of $\mathrm{NF}-\kappa \mathrm{Bp} 65$, c-IAP2 and caspase-3 expression in human cancer, this study used immunohistochemistry to determine their expression in cervical cancer and evaluate their significance in the diagnosis and prognosis of cervical cancer.

\section{Materials and methods}

Specimens. The present study included 44 patients with cervical cancer, with an average age of 51 years (range, 33-65). Specimens were formalin-fixed and paraffin-embedded. The cervical squamous cell carcinoma tissues were divided, according to World Health Organization (WHO) classification criteria and the status of cell differentiation, into three groups: 11 cases of grade I (well-differentiated), 14 cases of grade II (differentiated) and 19 cases of grade III (poorly differentiated or undifferentiated). The clinical stage of cervical squamous cell carcinoma was determined according to the International Federation of Gynecology and Obstetrics (FIGO) staging criteria. This study included 12 cases of stage I, 22 cases of stage II and 10 cases of stage III-IV. All patients did not receive 


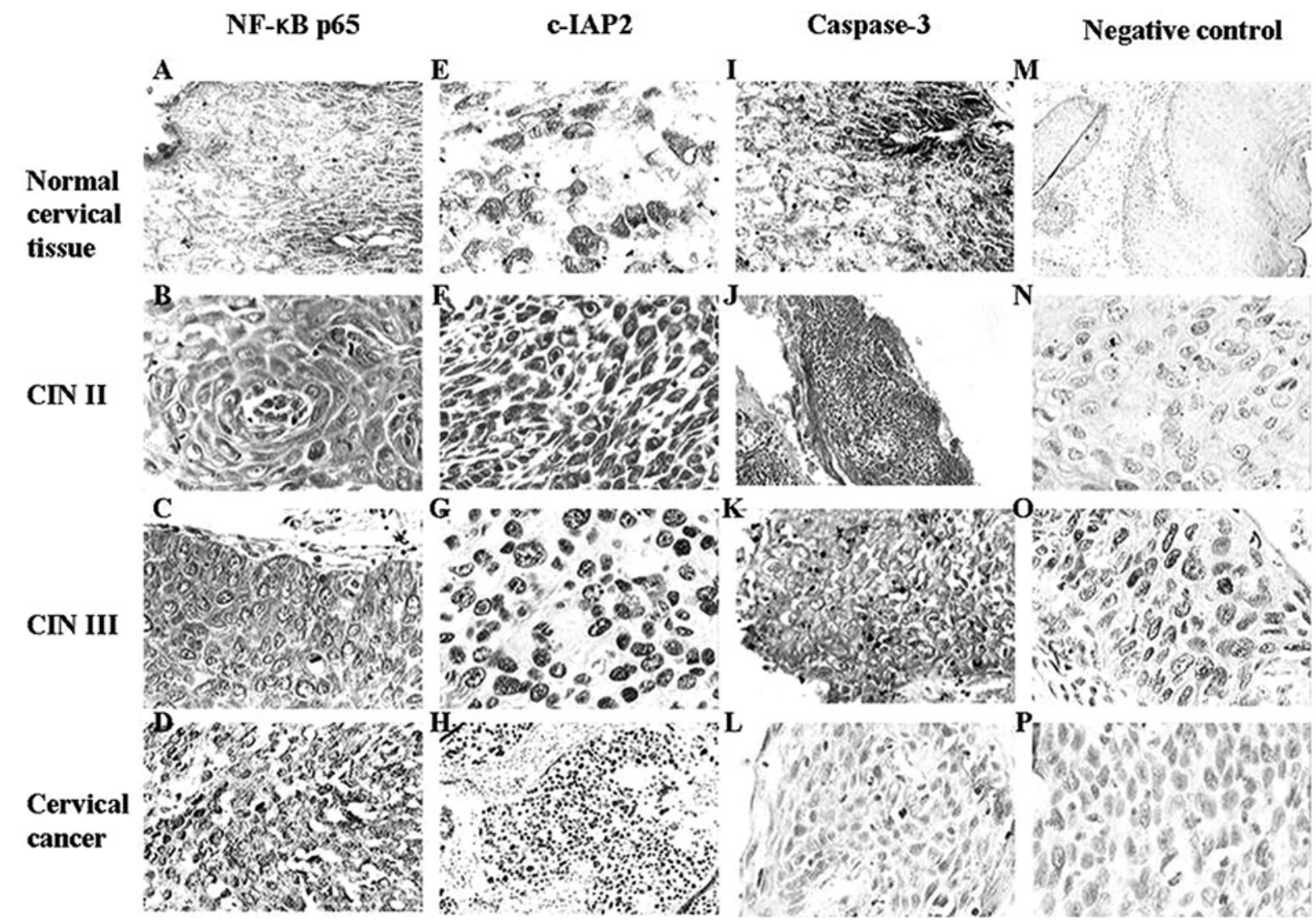

Figure 1. Immunohistochemical staining for expression of NF- $\kappa$ B p65, c-IAP2 and caspase-3. Expression of (A-D) NF- $\kappa$ B p65, (E-H) c-IAP2, (I-L) caspase-3 in normal cervix, CIN I, CIN III and cervical cancer, respectively. (M-P) Negative staining control. Each figure has original magnification, x100, with the exception of J, H and M. CIN, cervical intraepithelial neoplasia.

preoperative radiotherapy, chemotherapy, biological therapy or surgical treatment. The study population also consisted of 15 cases of CIN I (cervical squamous epithelium with mild dysplasia), 35 cases of CIN II-CIN III (moderate to severe squamous dysplasia) and 30 cases of normal cervical tissue. The stage I and II tumor specimens were retrieved from the surgical pathology archives of consecutive patients at the Nanyang First Hospital between 2005 and 2009. The stage III-IV specimens were obtained from outpatient biopsies at Nanyang First Hospital. All of the specimens were confirmed by histopathology.

The study was approved by the First Affiliated Hospital of Henan Scientific and Technological University. Consent was obtained from all patients who participated.

Immunohistochemistry. For the study, 4- $\mu \mathrm{m}$ serial sections of formalin-fixed, paraffin-embedded tissue were cut and mounted on positively charged glass slides. The following primary antibodies were applied at a working dilution of 1:50: mouse monoclonal antibody to NF- $\kappa$ B p65 (Santa Cruz Biotechnology, Santa Cruz, CA, USA), mouse monoclonal antibody to c-IAP2 (R\&D, CA, USA) and monoclonal antibody to caspase-3 (Beijing ZhongShan Biotech Co., Ltd, China). Immunohistochemical staining was conducted using a Streptavidin-Peroxidase (SP) kit purchased from Beijing ZhongShan Biotech Co., Ltd. The experimental procedures were performed according to the manufacturer's instructions; staining of a known positive tissue as the positive control and staining without the primary antibody as the negative control were carried out.

Evaluation. Analysis of the immunohistochemical staining results was performed under the supervision of senior pathologists. Immunohistochemical staining was evaluated using the semi-quantitative assay (2) and the positive results were determined based on yellow-brown staining of either the nucleus or cytoplasm. Counting for the scoring was performed by observation of a sample, with no less than 500 cells and more than 5 high power fields. Criteria for interpretation of the result, according to the color intensity of staining and the percentage of positive cells were: a) score of color intensity: 0 , colorless or blue; 1, light yellow; 2, brownish-yellow; 3, brown; b) score of the percentage of stained cells: $0, \leq 5 \%$ of cells; $1,6-20 \%$ of cells; $2,21-50 \%$ of cells, and $3, \geq 51 \%$ of cells; c) Overall score $=$ intensity score (a) $\mathrm{x}$ percentage of stained cells (b). The overall score was as follows: 0 points, negative, 1-3 points, '+', 4-6 points, '++', and 9 points, '+++'.

Statistical analysis. A $\chi^{2}$ test was used for clinical data analysis and correlation analysis was performed with Spearman's rank correlation coefficient. The experimental data were analyzed using SPSS 17.0 statistical software. For analysis of the results, $\mathrm{P}<0.05$ indicated a statistically significant difference and $\mathrm{P}<0.01$, a highly statistically significant difference. 
Table I. Expression of NF-кB p65, c-IAP2 and caspase-3 in cervical cancer, CIN and normal cervical tissue.

\begin{tabular}{|c|c|c|c|c|}
\hline & & $\mathrm{NF}-\kappa \mathrm{B}$ p $65^{\mathrm{a}}$ & c-IAP2 ${ }^{b}$ & Caspase- $3^{c}$ \\
\hline & Cases & Positive $(\%)$ & Positive (\%) & Positive (\%) \\
\hline Normal cervix & 30 & $3(10.0)$ & $5(16.7)$ & $23(77.0)$ \\
\hline $\mathrm{CIN}^{\mathrm{d}}$ & 50 & $17(34.0)$ & $22(44.0)$ & $18(36.0)$ \\
\hline Cervical cancer ${ }^{\mathrm{e}}$ & 44 & $30(68.0)$ & $34(77.0)$ & $7(16.0)$ \\
\hline
\end{tabular}

The groups were compared with normal cervix: ${ }^{\text {ad }} \chi^{2}=5.760, \mathrm{P}=0.016$; ${ }^{\mathrm{a}} \chi^{2}=24.439, \mathrm{P}=0.000$. ${ }^{\mathrm{bd}} \chi^{2}=6.265, \mathrm{P}=0.012 ;{ }^{\mathrm{be}} \chi^{2}=26.285, \mathrm{P}=0.000$. ${ }^{\mathrm{cd}} \chi^{2}=12.411, \mathrm{p}=0.000 ;{ }^{\mathrm{c}} \chi^{2}=27.317, \mathrm{P}=0.000 . \mathrm{CIN}$, cervical intraepithelial neoplasia.

Table II. Expression of NF-кB p65, c-IAP2 and caspase-3 in different grades of CIN.

\begin{tabular}{lcrrr}
\hline & & NF-kB p65 & c-IAP2 & Caspase-3 $^{\mathrm{b}}$ \\
\cline { 4 - 5 } & Cases & Positive $(\%)$ & Positive (\%) & Positive (\%) \\
\hline Normal cervix & 30 & $3(10.0)$ & $5(16.7)$ & $23(77.0)$ \\
CIN I $^{\mathrm{d}}$ & 15 & $2(13.3)$ & $3(20.0)$ & $9(60.0)$ \\
CIN II-III $^{\mathrm{e}}$ & 35 & $15(42.9)$ & $19(54.3)$ & $9(25.7)$ \\
\hline
\end{tabular}

The groups were compared with normal cervix: ${ }^{\mathrm{ad}} \chi^{2}=0.113, \mathrm{P}=0.737 ;{ }^{\mathrm{a}} \chi^{2}=8.709, \mathrm{P}=0.003 ;{ }^{\mathrm{bd}} \chi^{2}=0.076, \mathrm{P}=0.783 ;{ }^{\mathrm{be}} \chi^{2}=9.816, \mathrm{P}=0.002$. ${ }^{\mathrm{cd}} \chi^{2}=1.352, \mathrm{P}=0.245 ;{ }^{\mathrm{ce}} \chi^{2}=16.779, \mathrm{P}=0.000 . \mathrm{CIN}$, cervical intraepithelial neoplasia.

\section{Results}

Expression of $N F-\kappa B$ p65, c-IAP2 and caspase-3 in cervical cancer, CIN and normal cervical tissues. Representative results of the immunohistochemical staining are shown in Fig. 1. The three markers were present in the nucleus and cytoplasm. The cytoplasmic staining of $\mathrm{NF}-\kappa \mathrm{B}$ p 65 was stronger than that in the nucleus, while c-IAP2 exhibited a more intense nuclear staining. Caspase-3 revealed a dominant cytoplasmic staining pattern, similar to the pattern of $N F-\kappa B$. As shown in Table I, the percentage of positive-stained tissues for NF- $\kappa \mathrm{B}$ p65 was 68 (30/44), $34(17 / 50)$ and $10 \%$ (3/30) in cervical cancer, CIN and normal cervical tissue, respectively. The positive percentage of c-IAP2 was $77(34 / 44)$ and 44\% (22/50) in cervical cancer and CIN, respectively, which revealed statistical differences $(\mathrm{P}<0.05)$ compared with $16.7 \%$ percent positive $(5 / 30)$ in normal cervix (Table I). In contrast to the expression pattern of NF- $\mathrm{kB}$ p 65 and c-IAP2, the percentages of positive-stained tissues for caspase-3 were 16 (7/44), 36 $(18 / 50)$, and $77 \%(23 / 30)$ in cervical cancer, CIN and normal cervical tissue, respectively (Table I).

Expression of $N F-\kappa B$ p65, c-IAP2 and caspase-3 in different grades of CIN. The positive percentages of NF- $\kappa \mathrm{B}$ p 65 and c-IAP2 were 13.3 (2/15), 42.9 (15/35), 20 (3/15) and 54.3\% (19/35) in cervical mild squamous dysplasia (CIN I) and moderate to severe squamous dysplasia (CIN II-CIN III), respectively (Table II). The positive percentages of $\mathrm{NF}-\kappa \mathrm{B}$ p65 and c-IAP2 in CIN I were not significantly different from those in normal cervix $(\mathrm{P}>0.05)$. By contrast, the posi- tive percentage of NF- $\mathrm{NB}$ p65 and c-IAP2 in CIN II and CIN III were shown to be different, compared with those in normal cervical tissue $(\mathrm{P}<0.05)$, and the positive percentage of caspase-3 was 60 (9/15) and 25.7\% (9/35) in CIN I and CIN II-CIN III, respectively (Table II). The statistical analysis, as shown in Table II, revealed that the positive percentage of caspase-3 in CIN I was no different from that in normal cervix (P>0.05), but caspase-3 expression in CIN II-CIN III showed a difference from normal cervix $(\mathrm{P}<0.05)$.

Expression of NF- $\kappa B$ p65, c-IAP2 and caspase-3 in different stages and histological grades of cervical cancer, as well as in metastatic lymph nodes. The statistical analysis did not show a difference in NF- $\mathrm{NB}$ p65, c-IAP2 and caspase-3 expression in the various clinical stages of cervical cancer $(\mathrm{P}>0.05)$, as shown in Table III. However, the expression levels of NF- $\mathrm{B}$ p65 and c-IAP2 in poorly differentiated cancer and metastatic lymph nodes were higher than those in well-differentiated tumors and non-metastatic lymph nodes $(\mathrm{P}<0.05)$. Caspase-3 expression was lower in well-differentiated tumors and non-metastatic lymph nodes $(\mathrm{P}<0.05)$, which was inversely correlated with the expression pattern of NF-kB p65 and c-IAP2 (Table III).

Correlation between NF- $\kappa B$ p65, c-IAP2 and caspase-3 expression in cervical cancer. The statistical analysis, as shown in Table IV, revealed that NF- $\mathrm{B}$ p 65 and c-IAP2 expression in cervical cancer was positively correlated ( $\mathrm{rs}=0.561, \mathrm{P}=0.000$ ), while caspase- 3 expression in cervical cancer was negatively correlated with that of NF- $\kappa \mathrm{B}$ p65 and c-IAP2 ( $\mathrm{rs}=0.503$, $\mathrm{P}=0.000 ; \mathrm{rs}=0.677, \mathrm{P}=0.000$, respectively) (Table V). 
Table III. Expression of NF-kB p65, c-IAP2 and caspase-3 in different clinical stages and histological grades of cervical cancer as well as in metastatic lymph nodes.

\begin{tabular}{|c|c|c|c|c|}
\hline & & NF-кB p65 & c-IAP2 ${ }^{\mathrm{b}}$ & Caspase- $3^{c}$ \\
\hline & Cases & Positive (\%) & Positive (\%) & Positive (\%) \\
\hline Clinical stages & & & & \\
\hline I & 12 & $8(67.0)$ & $10(83.0)$ & $2(17.0)$ \\
\hline II & 22 & $15(68.2)$ & $16(73.0)$ & $3(14.0)$ \\
\hline III-IV & 10 & $7(70.0)$ & $8(80.0)$ & $2(20.0)$ \\
\hline Differentiation & & & & \\
\hline High & 11 & $4(36.4)$ & $5(45.5)$ & $5(45.5)$ \\
\hline Intermediate & 14 & $11(78.6)$ & $12(85.7)$ & $1(7.1)$ \\
\hline Low & 19 & 15 (78.9) & $17(89.5)$ & $1(5.3)$ \\
\hline Metastatic lym & & & & \\
\hline Negative & 20 & $10(50.0)$ & $12(60.0)$ & $5(25.0)$ \\
\hline Positive & 14 & $12(85.7)$ & 13 (92.9) & $0(0.0)$ \\
\hline
\end{tabular}

Table IV. Correlation analysis of NF- $\kappa \mathrm{B}$ p65 and c-IAP2 expression in cervical cancer.

\begin{tabular}{lcccc}
\hline & \multicolumn{4}{c}{ c-IAP2 } \\
\cline { 2 - 5 } NF-кB p65 & - & + & ++ & +++ \\
\hline- & 8 & 3 & 1 & 2 \\
+ & 1 & 3 & 4 & 3 \\
++ & 1 & 2 & 3 & 3 \\
+++ & 0 & 4 & 3 & 3 \\
\hline
\end{tabular}

\section{Discussion}

$\mathrm{NF}-\kappa \mathrm{B}$ is a nuclear transcription factor and is widely expressed in eukaryotic cells, and is involved in a variety of gene-specific transcription pathways. The NF- $\kappa \mathrm{B}$ family is composed of polypeptide subunits including Rel and NF- $\mathrm{\kappa B}$. The NF- $\mathrm{KB}$ subfamily contains p50/p105 (NF-kB1) and p52/

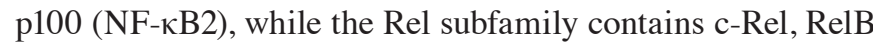
and RelA (p65) (3). Members of each subfamily are capable of forming homodimers or heterodimers. In mammals, the most common form of heterodimer is p50/RelA, or the trimer complex formed with its inhibitory protein IкB (ІкB- $\alpha$, I $\mathrm{K} B-\beta)$. Under resting conditions, the trimer complex as an inactive form exists in the cytoplasm of various types of cells. A variety of activation factors induce protein phosphorylation of I $\kappa \mathrm{B}$ and its subsequent degradation, leading to the release of the NF- $\mathrm{KB}$ dimer, followed by translocation of nucleus and sequence-specific DNA binding of target genes, thereby activating the transcription of target genes. In recent years, the overexpression and aberrant activation of NF- $\kappa \mathrm{B}$ has been
Table V. Correlation analysis of expression of NF- $\mathrm{kB}$ p65, c-IAP2 and caspase-3 in cervical cancer.

\begin{tabular}{lccccc}
\hline & \multicolumn{2}{c}{$\mathrm{NF- \kappa B} \mathrm{p65^{ \textrm {a } }}$} & & \multicolumn{2}{c}{$\mathrm{c}-\mathrm{IAP}_{2}{ }^{\mathrm{b}}$} \\
\cline { 2 - 3 } Caspase-3 & +-+++ & - & & +-+++ & - \\
\hline+-+++ & 1 & 6 & & 7 \\
- & 29 & 8 & & 34 & 6 \\
\hline
\end{tabular}

reported in head and neck squamous cell carcinoma, melanoma, prostate, cervical, non-small cell lung and colon cancer, and other human tumors. Our study found that the positive percentage of NF- $\mathrm{KB}$ p 65 was $68 \%$ in cervical cancer, but only $10 \%$ of cells were positive in normal cervix. There was a significant difference in NF- $\mathrm{KB}$ p65 expression between these two groups. Our results are consistent with the reports of Nair et al (4), suggesting that the abnormal expression of NF- $\mathrm{KB}$ p65 in cervical cancer may be important in cervical oncogenesis and the development of cervical cancer. However, expression of NF- $\mathrm{KB}$ p65 in different clinical stages of cervical cancer did not show a difference, but its expression in poorly differentiated squamous cell carcinoma was higher than that in the well-differentiated tumors. The expression of NF- $\mathrm{kB}$ p 65 in metastatic lymph nodes was higher than that in non-metastatic lymph nodes, suggesting that NF- $\kappa \mathrm{B}$ may be involved in the growth and metastasis of invasive cervical cancer.

c-IAP2 is a key anti-apoptotic factor, belonging to the inhibitor of apoptosis protein (IAPs) family. Apoptosis is sufficiently induced by knockout of the gene encoding c-IAP2. Overexpression of c-IAP2 has been found in a variety of human tumors and is correlated with drug resistance and recurrence of tumors, whereas, its expression in normal tissues is weak in the 
majority of cases. Cho et al (5) reported that c-IAP2 is overexpressed in ovarian cancer and related to tumor development. In their report, the expression of c-IAP2 was also detected in the normal ovary, where apoptosis is thought to play an important role in ovulation. Volp et al (6) found a marked correlation between two events in colon cancer, i.e., the induction of apoptosis and inhibition of HMGB1 and c-IAP2 expression. The underlying mechanisms are that overexpression of HMGB1 promotes $\mathrm{NF}-\kappa \mathrm{B}$ activity, thereby leading to co-overexpression of c-IAP2, a target gene of NF-кB. Varfolomeev et al (7) found that the c-IAP2/MALT1 fusion protein, which is caused by $\mathrm{t}(11$, 18) (q21; q21) translocation, may activate the NF- $\kappa B$ pathway, resulting in malignant transformation and development of lymphoma. In this study, the c-IAP2 expression level showed no significant difference in various clinical stages of cervical cancer. However, its expression level gradually increased from normal cervix to CIN, then to cervical cancer. With decreased histological grade of cervical cancer, c-IAP2 expression gradually increased. Notably, c-IAP2 expression in metastatic lymph nodes was higher than those in non-metastatic lymph nodes. All the differences were statistically significant, suggesting that c-IAP2 is important in oncogenesis, invasion and metastasis of cervical cancer.

Caspase-3 is the most important member of the caspase family and is widely expressed in normal human tissues and various tumor tissues. The majority of caspase family members are promoters or effectors of apoptosis, playing important roles in the process of apoptosis (8). Normal cells contain only a small amount of caspases, which exist in the form of inactive zymogens. Once caspases are activated, they have been transformed to proteases with enzyme catalytic activity and are capable of cleaving a number of substrate proteins, thereby resulting in apoptosis (9). The death receptor pathway of apoptosis, also known as the extrinsic pathway of apoptosis, is activated by binding of the death receptor to its corresponding ligand. Activation of the extrinsic pathway of apoptosis induces serial caspase activation, such as the initiator caspase, caspase-8 (10), and the effector caspases, caspase-3 and -7, (11) through the downstream signaling cascades, eventually leading to apoptosis. Caspases are inactive in the process of their synthesis and storage under normal circumstances. Zymogen activation of caspases induced by a variety of pro-apoptotic stimuli is the key step for promoting caspases in a cascade reaction during apoptosis, in which caspase- 3 activation is the most critical step. Our study found that the percentage of positive caspase-3 expression was significantly reduced in cervical cancer, compared with that in normal cervix and CIN. However, its expression was no different in various clinical stages of cervical cancer. This result is consistent with the findings of Zanotti et al (12), suggesting that a decreased caspase-3 expression is associated with the inhibition of apoptosis in cervical oncogenesis and the development of cervical cancer. Furthermore, our study found that caspase-3 expression in well-differentiated tumors was significantly higher than those in poorly differentiated ones. Its expression in non-metastatic lymph nodes was higher than those in metastatic lymph nodes, indicating that a low expression of caspase- 3 is closely related to the differentiation degree and invasion/metastasis of cervical cancer. The less the cancer cells were differentiated, the more inhibition of apoptosis occurred, consequently leading to a higher degree of malignancy of cancer cells, as well as tumor cell invasion and metastasis.

The molecular mechanisms underlying the role of $\mathrm{NF}-\kappa \mathrm{B}$ in the tumor have not yet been fully understood. In the resting cells, $\mathrm{NF}-\kappa \mathrm{B}$ is present as homo- or heterodimers associated with its inhibitory protein $\mathrm{I} \kappa \mathrm{B}$ family members in the cytoplasm, which prevent $\mathrm{NF}-\kappa \mathrm{B}$ activation. When cells are stimulated by a variety of stimuli, dissociation of $\mathrm{I} \kappa \mathrm{B}$ from the $\mathrm{NF}-\kappa \mathrm{B}$ dimer leads to nuclear translocation of $\mathrm{NF}-\kappa \mathrm{B}$ and sequential target gene transactivation. $\mathrm{NF}-\kappa \mathrm{B}$ is known to be activated by at least two different pathways, i.e., the classical and the alternative pathways. Activation of the classical pathway depends on $\mathrm{I} \kappa \mathrm{K} \beta$ activity, while activation of the alternative pathway relies on I $\kappa \alpha \alpha$ activity. Activation of $\mathrm{NF}-\kappa \mathrm{B}$ is involved in tumor development, angiogenesis and metastasis, thereby promoting invasion and spread of tumor cells. NF- $\kappa \mathrm{B}$ plays a role in promoting tumor proliferation through induction of anti-apoptotic gene expression, such as c-IAP2 and other anti-apoptotic factors. The mechanism underlying c-IAP2-mediated inhibition of apoptosis depends on the caspase family. Apoptosis is actually the irreversible process of substrate cleavage mediated by caspases. The direct association of c-IAP2 with caspase-3, -7 and -9 attenuates the apoptotic signal transduction, thereby inhibiting the incidence of apoptosis and promoting tumorigenesis. However, c-IAP2 is also involved in the inhibition of procaspase-9 zymogen activation and the sequential zymogen activation of pro-caspase- 3 and -7 , as well as in the prevention of further activation of caspase -9 caused by a positive feedback mechanism from caspase-3 activation. In addition, the carboxyl-terminal RINGfinger motif of c-IAP2 possesses intrinsic ubiquitin ligase activity. When exposed to various apoptotic stimuli, the RING domain of c-IAP2 mediates ubiquitin ligase activity for selfubiquitination and ubiquitination of the associated caspases, thereby regulating their protein degradation levels (13). Further study found that NF- $\kappa \mathrm{B}$ is required for c-IAP2-mediated inhibition of TNF- $\alpha$-induced apoptosis and NF- $\kappa$ B plays a crucial role in the anti-apoptotic function of IAPs, suggesting that a prompt inhibition of c-IAP2 expression corrects the imbalances between pro-apoptotic and anti-apoptotic mechanisms and restore programmed cell death. Our study found that the expression of NF- $\mathrm{BB}$ p 65 and c-IAP2 is positively correlated with cervical cancer and negatively correlated with caspase-3 expression in the tumor, suggesting that $\mathrm{NF}-\kappa \mathrm{B}$ promotes cervical cancer development through the possible mechanism of c-IAP2 gene transcriptional regulation, by which c-IAP2 protein is overexpressed and caspase-3 activity is inhibited. The central regulatory roles of $\mathrm{NF}-\kappa \mathrm{B}$ in the immune, inflammatory and other processes have been confirmed by a number of research institutions. Recently more and more attention has been paid to the roles of $\mathrm{NF}-\kappa \mathrm{B}$ in tumorigenesis and tumor development. In this study, we found that the overexpression of $\mathrm{NF}-\kappa \mathrm{B}$ is positively correlated with those of c-IAP2 in cervical cancer, while overexpression of NF- $\mathrm{B}$ and c-IAP2 in cervical cancer is negatively correlated with decreased caspase-3 expression in tumors. All of our observed results revealed the clinicopathological significance of NF- $\kappa \mathrm{B}$ p 65 , c-IAP2 and caspase-3 expression in cervical cancer and their potential value as biomarkers for the diagnosis and prognosis of cervical cancer. 


\section{References}

1. Mazumder S, Plesca D and Almasan A: Caspase-3 activation is a critical determinant of genotoxic stress-induced apoptosis. Methods Mol Biol 414: 13-21, 2008.

2. Xia KD, Chen XM, Lin QA, Dong HY, Chen S and Zhang LF: Correlation of expression of nuclear factor kappaB, inhibitor protein kappaB, and $\mathrm{Bcl}-2$ in cervical cancer to human papillomavirus infection. Ai Zheng 24: 1350-1353, 2005.

3. Gilmore TD: Introduction to NF- $\mathrm{BB}$ : players, pathways, perspectives. Oncogene 25: 6680-6684, 2006.

4. Nair A, Venkatraman M, Maliekal TT, Nair B and Karunagaran D: NF- $\kappa \mathrm{B}$ is constitutively activated in high-grade squamous intraepithelial lesions and squamous cell carcinomas of the human uterine cervix. Oncogene 22: 50-58, 2003.

5. Cho CH CS, Baek WK, Kwon KY and Bae IS: Expression analysis of c-IAP2 in ovarian carcinomas. Korean J Obstet Gynecol 44: 6, 2001.

6. Volp K, Brezniceanu ML, Bosser S, Brabletz T, Kirchner T, Gottel D, Joos S and Zornig M: Increased expression of high mobility group box 1 (HMGB1) is associated with an elevated level of the antiapoptotic c-IAP2 protein in human colon carcinomas. Gut 55: 234-242, 2006.

7. Varfolomeev E, Wayson SM, Dixit VM, Fairbrother WJ and Vucic D: The inhibitor of apoptosis protein fusion c-IAP2. MALT1 stimulates NF- $\kappa$ B activation independently of TRAF1 AND TRAF2. J Biol Chem 281: 29022-29029, 2006.

8. Stepien A, Izdebska M and Grzanka A: The types of cell death, Postepy Hig Med Dosw 61: 420-428, 2007.

9. Bozonet SM, Scott-Thomas AP, Nagy P and Vissers MC: Hypothiocyanous acid is a potent inhibitor of apoptosis and caspase 3 activation in endothelial cells. Free Radic Biol Med 49: 1054-1063, 2010.

10. Zhao Z, Yang P, Eckert RL and Reece EA: Caspase-8: a key role in the pathogenesis of diabetic embryopathy. Birth Defects Res B Dev Reprod Toxicol 86: 7, 2009.

11. Odonkor CA and Achilefu S: Modulation of effector caspase cleavage determines response of breast and lung tumor cell lines to chemotherapy. Cancer Invest 27: 417-429, 2009.

12. Zanotti S, Fisseler-Eckhoff A and Mannherz HG: Changes in the topological expression of markers of differentiation and apoptosis in defined stages of human cervical dysplasia and carcinoma. Gynecol Oncol 89: 376-384, 2003.

13. Varfolomeev E and Vucic D: (Un)expected roles of c-IAPs in apoptotic and NFKB signaling pathways. Cell Cycle 7: 1511-1521, 2008. 